\title{
CSF hypocretin-1 levels in narcolepsy, Kleine-Levin syndrome, and other hypersomnias and neurological conditions
}

\author{
Y Dauvilliers, CR Baumann, B Carlander, M Bischof, T Blatter, M Lecendreux, F Maly, A Besset, \\ J Touchon, M Billiard, M Tafti, CL Bassetti
}

J Neurol Neurosurg Psychiatry 2003;74:1667-1673

See end of article for authors' affiliations

Correspondence to:

Dr Yves Dauvilliers, Service de Neurologie B, Hôpital Gui-de-Chauliac, 80 avenue Augustin Fliche, 34295 Montpellier cedex 5, France; ydauvilliers@ yahoo.fr

Received 7 March 2003 Revised version received 14 May 2003

Accepted for publication 17 May 2003

\begin{abstract}
Objective: To determine the role of CSF hypocretin-1 in narcolepsy with and without cataplexy, KleineLevin syndrome (KLS), idiopathic and other hypersomnias, and several neurological conditions. Patients: 26 narcoleptic patients with cataplexy, 9 narcoleptic patients without cataplexy, 2 patients with abnormal REM-sleep-associated hypersomnia, 7 patients with idiopathic hypersomnia, 2 patients with post-traumatic hypersomnia, 4 patients with KLS, and 88 patients with other neurological disorders. Results: 23 patients with narcolepsy-cataplexy had low CSF hypocretin-1 levels, while one patient had a normal hypocretin level (HLA-DQB1 ${ }^{*} 0602$ negative) and the other two had intermediate levels (familial forms). One narcoleptic patient without cataplexy had a low hypocretin level. One patient affected with post-traumatic hypersomnia had intermediate hypocretin levels. The KLS patients had normal hypocretin levels while asymptomatic, but one KLS patient (also affected with Prader-Willi syndrome) showed a twofold decrease in hypocretin levels during a symptomatic episode. Among the patients without hypersomnia, two patients with normal pressure hydrocephalus and one with unclear central vertigo had intermediate levels.

Conclusion: Low CSF hypocretin-1 is highly specific (99.1\%) and sensitive (88.5\%) for narcolepsy with cataplexy. Hypocretin ligand deficiency appears not to be the major cause for other hypersomnias, with a possible continuum in the pathophysiology of narcolepsy without cataplexy and idiopathic hypersomnia. However, partial hypocretin lesions without low CSF hypocretin-1 consequences cannot be definitely excluded in those disorders. The existence of normal hypocretin levels in narcoleptic patients and intermediate levels in other rare aetiologies needs further investigation, especially for KLS, to establish the functional significance of hypocretin neurotransmission alterations.
\end{abstract}

$\mathrm{N}$ arcolepsy is characterised by two major symptoms, excessive daytime sleepiness (EDS) and cataplexy and other manifestations of abnormal rapid eye movement (REM) sleep. ${ }^{1}$ Although narcolepsy has been known as a distinct clinical entity for more than a century, ${ }^{2}$ it was only recently that a major discovery in its pathophysiology was made through the identification of orexin/hypocretin deficiency. $^{3-7}$ While a single mutation in hypocretin genes has been found in one patient with atypical narcolepsy, undetectable levels of CSF hypocretin-1 were reported in most patients with sporadic narcolepsy ${ }^{7-9}$ and a dramatic decrease of hypocretin neurones in a few postmortem narcoleptic brains. ${ }^{5}{ }^{6}$ Specificity and sensitivity of low CSF hypocretin- 1 levels for human narcolepsy-cataplexy are very high. ${ }^{89}$ Among the large narcoleptic populations reported, only a few patients had normal CSF hypocretin-1 levels and most were HLA DQB1*0602 negative, without cataplexy, or with a familial form of narcolepsy. ${ }^{89}$ The role of the hypocretin system in other hypersomnia disorders is uncertain. Few studies reported normal CSF hypocretin-1 levels in most narcoleptic patients without cataplexy, in idiopathic hypersomnia, and in almost all other neurological disorders except for the Guillain-Barré syndrome and other rare conditions. ${ }^{8-14}$ Recently, a study reported CSF hypocretin deficiency in all patients affected with narcolepsy with or without cataplexy, and in idiopathic hypersomnia. ${ }^{15}$ The mechanism of differences between CSF hypocretin-1 levels and narcolepsy phenotype (with or without cataplexy, sporadic or familial, and whether HLA-associated or not) among studies remains controversial.
The hypocretin system might also be involved in other patients with hypersomnia. Among these, myotonic dystrophy $^{1617}$ and Prader-Willy syndrome ${ }^{18}$ share the presence of sleep onset REM periods (SOREMPs) during a multiple sleep latency test (MSLT) with narcolepsy. The mechanism of hypersomnia with SOREMPs first suggested a central nervous system dysfunction; however the excessive daytime sleepiness may be in relation to sleep fragmentation that is secondary to frequent sleep-disordered breathing in those pathologies. ${ }^{16}{ }^{18}$ Sleep apnoea syndrome could also share the presence of sleep onset REM periods with narcolepsy ${ }^{19}$; however the mechanisms involved may be in relation to sleep deprivation only. Also, because the hypocretinergic neurotransmission plays a significant role in the regulation of both feeding and sleep-wake cycles, ${ }^{20}$ Kleine-Levin syndrome (KLS), which is characterised by recurrent hypersomnia and abnormal feeding behaviour, ${ }^{21-23}$ might represent a model disorder for hypocretin system malfunction. In this study, we investigated the CSF hypocretin-1 levels in different disorders with and without hypersomnia or feeding behaviour abnormalities and looked for phenotypic correlations with low hypocretin-1 levels.

Abbreviations: EDS, excessive daytime sleepiness; ICSD, international classification of sleep disorders; KLS, Kleine-Levin syndrome; MSLT, multiple sleep latency test; PLM, periodic leg movements; RDI, respiratory disturbances index; SOREMP, sleep onset REM period 


\section{PATIENTS AND METHODS}

One hundred and thirty eight unrelated patients were included. Three European collaborating centres contributed with 67 (YD, BC, JT, MBil, Montpellier, France, Centre 1), 69 (CRB, MBis, TB, FM, CLB, Zurich, Switzerland, Centre 2), and 2 patients (ML, Paris, France, Centre 3). Informed consent of all patients was obtained before lumbar puncture.

Sleep disorders were diagnosed clinically and using sleep studies. Patients were classified based on their primary ICSD (international classification of sleep disorders) diagnosis, ${ }^{21}$ blinded of CSF hypocretin-1 results. Demographic, clinical, polygraphic and HLA typing data are described in tables $1-4$. The cataplexy severity was assessed on a scale from 1 to 5 , where: $1=$ one cataplectic attack per year or fewer; $2=$ more than one attack per year but less than one per month; $3=$ more than one attack per month but less than one per week; $4=$ more than one per week but less than one per day; and $5=$ severe cases with at least one cataplectic attack per day. A mean respiratory disturbances index (RDI) including oesophageal pressure monitoring of less than 5 per hour and a periodic leg movements (PLM) index during sleep of less than 5 per hour were necessary to assess the primary sleep disorders diagnosis (-that is, narcolepsy without cataplexy, idiopathic hypersomnia, post-traumatic hypersomnia) except for narcolepsy with cataplexy.

Twenty-six (15 males and 11 females) narcoleptic patients with clear-cut cataplexy were included: three reported a positive family history of narcolepsy-cataplexy (major form) and three others a positive family history of isolated recurrent daytime naps or lapses into sleep (minor form) ${ }^{24} 25$ and all except one were HLA DQB1*0602 positive (table 1).
Nine (five males and four females) narcoleptic patients without cataplexy were included: two reported a positive family history of narcolepsy without cataplexy and all except one were HLA DQB1*0602 positive (table 2). Two other patients with SOREMP-associated hypersomnia were included: one with sleep apnoea syndrome and one with genetically confirmed myotonic dystrophy (expansion of 133 CTG repeat $)^{26}$ and HLA DRB1*11/13 and DQB ${ }^{*} 0301 / 0603$ alleles (table 2 ). His sister was also affected with myotonic dystrophy and SOREMP-associated hypersomnia. Seven patients (five males and two females) with polysymptomatic idiopathic hypersomnia ${ }^{212728}$ were included: one reported a positive family history of hypersomnia and three were HLA DQB1*0602 positive (table 2 ). Two more patients were affected with post-traumatic hypersomnia ${ }^{29}{ }^{30}$ (table 2 ). The clinical history of case 1 revealed a severe traumatic brain injury with coma lasting for several days and the occurrence of excessive daytime sleepiness three months after. Brain MRI showed several small bilateral frontal and bilateral posttraumatic lesions without hypothalamic lesions. The second patient (case 2) had a less severe traumatic brain injury without coma or lesions, as revealed by MRI, with the occurrence of excessive daytime sleepiness two months after the trauma.

Four patients affected with Kleine-Levin syndrome were included (table 3 ). The diagnostic criteria were clinical and required at least $(a)$ recurrent episodes of hypersomnia and (b) cognitive and mood disturbances present only during symptomatic episodes. ${ }^{21-23}$ The presence of the two other symptoms, hyperphagia and hypersexuality (in at least one symptomatic episode) was not necessary and was observed in

Table 1 CSF hypocretin-1 in narcoleptic patients with cataplexy

\begin{tabular}{|c|c|c|c|c|c|c|c|c|c|c|c|c|c|c|c|c|c|}
\hline Subjects & Centre & Age & Sex & BMI & $\begin{array}{l}\text { Age at } \\
\text { onset } \\
\text { of EDS }\end{array}$ & $\begin{array}{l}\text { Age at } \\
\text { onset of } \\
\text { cataplexy }\end{array}$ & $\begin{array}{l}\text { Family } \\
\text { history }\end{array}$ & $\mathbf{E}$ & C & $\mathrm{HH}$ & SP & MSLT & SOREMP & $\begin{array}{l}\text { REM } \\
\text { latency }\end{array}$ & $\begin{array}{l}\text { HLA } \\
\text { DQB } 1 * 0602\end{array}$ & $\mathbf{T}$ & Нуро- 1 \\
\hline 1 & 1 & 36 & $\mathrm{~F}$ & 23.8 & 28 & 28 & 0 & 18 & 3 & + & + & 4 & 3 & 61.5 & + & - & 85 \\
\hline $2^{*}$ & 1 & 55 & $\mathrm{~F}$ & 31.6 & 46 & 46 & 0 & 24 & 5 & + & - & 3.6 & 2 & 17 & + & + & $<10$ \\
\hline 3 & 1 & 57 & $M$ & 21.5 & 51 & 53 & 0 & 23 & 3 & 0 & + & 4.6 & 5 & 63 & + & - & 46 \\
\hline 4 & 1 & 52 & $M$ & 25.9 & 15 & 15 & $N$ & 21 & 5 & + & + & 4 & 5 & 129 & + & + & $<10$ \\
\hline 5 & 1 & 33 & $M$ & 26.8 & 16 & 20 & 0 & 23 & 4 & 0 & + & 2 & 4 & 6 & + & + & 79 \\
\hline 6 & 1 & 39 & $M$ & 27.5 & 18 & 27 & 0 & 14 & 5 & + & + & 4 & 4 & 2 & + & - & $<10$ \\
\hline 7 & 1 & 19 & $\mathrm{~F}$ & 27.7 & 4 & 17 & 0 & 12 & 3 & + & - & 1.7 & 4 & 3.5 & + & - & 51 \\
\hline 8 & 1 & 20 & $\mathrm{~F}$ & 26.4 & 10 & 13 & 0 & 24 & 1 & 0 & - & 2.25 & 3 & 68 & + & - & 38 \\
\hline 9 & 1 & 22 & $M$ & 22.6 & 12 & 20 & $\mathrm{H}$ & 17 & 4 & + & + & 6.4 & 3 & 95.5 & + & - & 126 \\
\hline 10 & 1 & 17 & $M$ & 17.2 & 7 & 9 & 0 & 18 & 5 & + & - & 0.8 & 5 & 0 & + & + & $<10$ \\
\hline 11 & 1 & 55 & $M$ & 26.1 & 40 & 50 & 0 & 24 & 4 & + & - & 6.1 & 4 & 3.5 & + & + & $<10$ \\
\hline 12 & 1 & 35 & $M$ & 29.8 & 20 & 19 & 0 & 18 & 5 & + & + & 1.5 & 4 & 7 & + & + & $<10$ \\
\hline 13 & 1 & 55 & $M$ & 26.8 & 20 & 24 & $\mathrm{H}$ & 18 & 3 & 0 & - & 6.5 & 4 & 0.5 & + & - & 25 \\
\hline 14 & 1 & 13 & $\mathrm{~F}$ & 13.7 & 4 & 4 & 0 & 18 & 5 & + & - & 2.6 & 4 & 40 & + & - & 14 \\
\hline 15 & 1 & 29 & $M$ & 24.8 & 29 & 29 & 0 & 23 & 5 & + & + & 3 & 5 & 0 & + & + & $<10$ \\
\hline 16 & 1 & 23 & $\mathrm{~F}$ & 23.4 & 20 & 20 & $\mathrm{H}$ & 24 & 5 & + & + & 1.2 & 5 & 0 & + & + & $<10$ \\
\hline 17 & 1 & 26 & $\mathrm{~F}$ & 32.6 & 1 & 12 & $N$ & 24 & 5 & + & + & 1.6 & 5 & 69.5 & + & + & $<10$ \\
\hline $18+$ & 1 & 51 & $\mathrm{~F}$ & 21.8 & 25 & 47 & 0 & 14 & 5 & + & + & 14.6 & 2 & 22 & - & + & 271 \\
\hline 19 & 1 & 26 & $\mathrm{~F}$ & 21.4 & 11 & 11 & 0 & 19 & 5 & - & + & 2.6 & 3 & na & + & + & $<10$ \\
\hline 20 & 2 & 64 & $M$ & 26.3 & 43 & 44 & na & 19 & 5 & - & - & 4.5 & 4 & 5 & + & + & $<10$ \\
\hline 21 & 2 & 34 & $M$ & 25.8 & 25 & 23 & 0 & 18 & 3 & + & + & 4.9 & 4 & 6 & + & + & $<10$ \\
\hline 22 & 2 & 21 & $M$ & 20.2 & 12 & 14 & 0 & 16 & 4 & + & - & 5.3 & 5 & 15 & + & - & $<10$ \\
\hline 23 & 2 & 52 & M & 28.1 & 24 & 24 & 0 & 20 & 4 & - & - & 4 & 3 & 28 & + & + & $<10$ \\
\hline 24 & 2 & 29 & M & 27.5 & 17 & 18 & 0 & 16 & 4 & + & - & 6 & 4 & 7 & + & - & $<10$ \\
\hline 25 & 2 & 18 & $\mathrm{~F}$ & 18.0 & 14 & 12 & 0 & 17 & 4 & - & + & 2.5 & 4 & 8 & + & - & 14 \\
\hline 26 & 2 & 37 & $\mathrm{~F}$ & 22.8 & 20 & 20 & $\mathrm{~N}$ & 17 & 3 & + & + & 4 & 3 & 10 & + & - & 145 \\
\hline \multicolumn{2}{|c|}{ Mean (SD) } & $\begin{array}{l}35.3 \\
(15.1)\end{array}$ & & $\begin{array}{l}24.6 \\
(4.4)\end{array}$ & $\begin{array}{l}20.5 \\
(13)\end{array}$ & $\begin{array}{l}23.8 \\
(13.5)\end{array}$ & & $\begin{array}{l}19.2 \\
(3.5)\end{array}$ & $\begin{array}{l}4.1 \\
(1.03)\end{array}$ & & & $\begin{array}{r}4.0 \\
(2.7)\end{array}$ & $\begin{array}{l}3.9 \\
(0.9)\end{array}$ & $\begin{array}{r}26.7 \\
(34.7)\end{array}$ & & & $\begin{array}{l}40.2 \\
(60.2)\end{array}$ \\
\hline $\begin{array}{l}\text { Centre 1: } \\
\text { F, female; } \\
\text { hallucinatic } \\
\text { Family hist } \\
\text { T, treatmen } \\
\text { *Status catc } \\
\text { tHLA DRB }\end{array}$ & $\begin{array}{l}\text { Montpell } \\
\text { M, male } \\
\text { on; SP, s } \\
\text { tory: 0, } \\
\text { nt: Psych } \\
\text { aplecticu } \\
1{ }^{*} 04 / 11\end{array}$ & $\begin{array}{l}\text { lier, Frar } \\
\text { e; BMI, b } \\
\text { sleep pa } \\
\text { none; N } \\
\text { nostimula } \\
\text { Us for se } \\
1 \text {-DQB } 1\end{array}$ & $\begin{array}{l}\text { nce; C } \\
\text { oody } r \\
\text { ralysi } \\
\text {, fami } \\
\text { ant or } \\
\text { veral }\end{array}$ & $\begin{array}{l}\text { Centre } \\
\text { mass i } \\
\text { is; Hyp } \\
\text { ily hist } \\
\text { antica } \\
\text { days }\end{array}$ & $\begin{array}{l}\text { Zurich, } \\
\text { lex; EDS } \\
\text { 1, level } \\
\text { y of nar } \\
\text { plectic } t \\
\text { en lumb }\end{array}$ & $\begin{array}{l}\text { Switzerland } \\
\text { excessive } \\
\text { of hypocreti } \\
\text { colepsy; } H, \\
\text { eatment at } \\
\text { ar puncture }\end{array}$ & $\begin{array}{l}\text { daytime } \mathrm{s} \\
\text { n-1 in the } \\
\text { family his } \\
\text { time of C } \\
\text { was mac }\end{array}$ & $\begin{array}{l}\text { leepines } \\
\text { CSF ( } p \\
\text { tory of } \\
\text { SF lumb } \\
\text { de }\end{array}$ & $\begin{array}{l}\text { s; E, Epv } \\
\mathrm{g} / \mathrm{ml}) ; \mathrm{n} \\
\text { yypersom } \\
\text { ar punct }\end{array}$ & $\begin{array}{l}\text { a, nc } \\
\text { nnia } \\
\text { ure }\end{array}$ & & $\begin{array}{l}\text { ale } \\
\text { ido }\end{array}$ & $\begin{array}{l}\text { cataple } \\
\text { child) }\end{array}$ & & at & & \\
\hline
\end{tabular}


Table 2 CSF hypocretin-1 in patients with narcolepsy without cataplexy, idiopathic and other hypersomnias

\begin{tabular}{|c|c|c|c|c|c|c|c|c|c|c|c|c|c|c|}
\hline Diagnosis & Centre & Age & Sex & $\begin{array}{l}\text { Age of } \\
\text { onset }\end{array}$ & $\begin{array}{l}\text { Family } \\
\text { history }\end{array}$ & $\mathbf{E}$ & C & $\mathrm{HH}$ & SP & MSLT & SOREMPs & RDI & $\begin{array}{l}\text { HLA } \\
\text { DQB 1*0602 }\end{array}$ & Нypo-1 \\
\hline \multicolumn{15}{|c|}{ Narcolepsy without cataplexy } \\
\hline $1^{*}$ & 1 & 18 & M & 14 & 0 & 17 & - & - & - & 7 & 2 & 3.4 & - & 346 \\
\hline 2 & 1 & 34 & $\mathrm{~F}$ & 8 & 0 & 16 & - & + & - & 3.3 & 2 & 0.7 & + & 597 \\
\hline 3 & 1 & 26 & $\mathrm{~F}$ & 16 & 0 & 19 & - & + & + & 13.8 & 1 & $<5$ & + & 100 \\
\hline 4 & 2 & 22 & M & 20 & 0 & 15 & - & + & - & 6.5 & 3 & 0.8 & + & 236 \\
\hline 5 & 2 & 31 & $M$ & 25 & 0 & 18 & - & + & + & 3 & 4 & 3.9 & + & 393 \\
\hline 6 & 2 & 50 & $M$ & 30 & na & 16 & - & + & - & 7 & 3 & 3.1 & + & 419 \\
\hline 7 & 2 & 37 & $\mathrm{~F}$ & 21 & $\mathrm{H}$ & 21 & - & + & + & 5.4 & 5 & 0.9 & + & 490 \\
\hline 8 & 2 & 39 & $\mathrm{~F}$ & 30 & $\mathrm{H}$ & 17 & - & + & - & 4 & 4 & 1.2 & + & 537 \\
\hline 9 & 2 & 37 & $M$ & 19 & 0 & 16 & - & + & - & 5.5 & 3 & 1.6 & + & 591 \\
\hline \multicolumn{15}{|c|}{ Idiopathic hypersomnia } \\
\hline 1 & 1 & 31 & $M$ & 31 & 0 & 19 & - & - & - & 4.4 & 1 & 1.1 & + & 609 \\
\hline 2 & 2 & 36 & $M$ & 26 & 0 & 14 & - & - & - & 5 & 0 & 1.4 & - & 204 \\
\hline 3 & 2 & 61 & $M$ & 21 & 0 & 17 & - & - & - & 5.2 & 0 & 2 & + & 327 \\
\hline 4 & 2 & 28 & $\mathrm{~F}$ & 22 & $\mathrm{H}$ & 16 & - & - & - & 4 & 0 & 1.1 & - & 342 \\
\hline 5 & 2 & 49 & $\mathrm{~F}$ & 37 & 0 & 15 & - & - & - & 6.4 & 0 & 1.8 & - & 400 \\
\hline 6 & 2 & 36 & $M$ & 30 & 0 & 17 & - & - & - & 7.1 & 0 & 3.5 & + & 421 \\
\hline 7 & 2 & 41 & $M$ & 35 & 0 & 14 & - & - & - & 4.2 & 0 & 0.9 & - & 1130 \\
\hline \multicolumn{15}{|c|}{ Post-traumatic hypersomnia } \\
\hline 1 & 2 & 29 & $M$ & 23 & 0 & 17 & - & - & - & 4.5 & 0 & 2 & - & 176 \\
\hline 2 & 2 & 40 & $M$ & 21 & 0 & 19 & - & - & - & 3 & 0 & 3.1 & + & 503 \\
\hline \multicolumn{15}{|c|}{ Sleep apnoea syndrome } \\
\hline 1 & 1 & 49 & M & 14 & 0 & 22 & - & + & - & 2.1 & 4 & 27.8 & - & 476 \\
\hline \multicolumn{15}{|c|}{ Myotonic dystrophy } \\
\hline 1 & 1 & 23 & $M$ & 19 & $\mathrm{H}$ & 18 & - & - & - & 6.4 & 3 & 5 & - & 401 \\
\hline
\end{tabular}

Centre 1: Montpellier, France; Centre 2: Zurich, Switzerland

$\mathrm{F}$, female; $\mathrm{M}$, male; E, Epworth sleepiness scale; HH, hypnagogic hallucination; SP, sleep paralysis; RDI, respiratory disturbances index including oesophageal pressure monitoring; Hypo-1, level of hypocretin-1 in the CSF (pg/ml); na, not available

Family history: $\mathrm{O}$, none; $\mathrm{H}$, family history of hypersomnia

* HLA DRB 1 *07/08-DQB 1 *02/04

three cases (table 3). Brain imaging, CSF cytology and protein levels were normal in all four patients. Patient 4 was however atypical because of an associated genetically confirmed Prader-Willi syndrome (methylation on 15q11q12), ${ }^{31}$ an association that was also previously described. ${ }^{32}$

Eighty-eight (38 males and 50 females) patients affected with well-defined neurological disorders were also included in this study (table 4). Patients were diagnosed by clinical, radiological, and laboratory investigations, and grouped into different categories based on their final diagnosis.

Five patients (two narcoleptic patients with cataplexy, two patients with idiopathic hypersomnia, one patient with posttraumatic hypersomnia) included in this series were also included in a previous report. ${ }^{13}$

CSF samples were collected between 9 am and $5 \mathrm{pm}$ and stored immediately at $-80^{\circ} \mathrm{C}$ until use (storage period $=2-$ 120 days). The current treatment in narcoleptic and other hypersomnia patients (anticataplectic or psychostimulant drugs) was recorded at the time of lumbar puncture. Lumbar puncture in the patients with Kleine-Levin syndrome was made during asymptomatic periods in cases 1, 2 and 3, and during both symptomatic and asymptomatic periods in case 4 (table 3 ).

\section{Hypocretin radioimmunoassay}

Hypocretin-1 (orexin-A) was determined at least in duplicate from CSF samples without prior extraction using ${ }^{125} \mathrm{I}$ radioimmunoassay kits from Phoenix Peptide, Inc, according to the manufacturer's prescriptions. The detection limit was $10 \mathrm{pg} / \mathrm{ml}$ and intra-assay variability was less than $10 \%$. The mean daytime hypocretin-1 levels in the CSF of 47 published healthy controls is 363.2 (16.3) pg/ml (range 224-653) for the direct assay. ${ }^{93}$ Hypocretin-1 CSF levels less than 110 pg/ $\mathrm{ml}$ were classified as low, between 110 and $200 \mathrm{pg} / \mathrm{ml}$ as intermediate and above $200 \mathrm{pg} / \mathrm{ml}$ as normal, as in Mignot et al. ${ }^{9}$

\section{Statistical analysis}

All results are reported as mean (standard deviation (SD)). For statistical analysis, the undetectable hypocretin level was arbitrarily set at $10 \mathrm{pg} / \mathrm{ml}$ (一that is, our detection limit). Between group comparisons were performed by analysis of variance. Tukey's test was used after a significant result. Correlation coefficients were calculated according to Pearson's method. Statistical significance was set at $\mathrm{p}<0.05$.

Table 3 CSF Hypocretin-1 in patients with Kleine-Levin syndrome

\begin{tabular}{|c|c|c|c|c|c|c|c|c|c|c|c|c|}
\hline Subjects & Centre & Age & Sex & $\begin{array}{l}\text { Age of } \\
\text { onset }\end{array}$ & $\begin{array}{l}\text { Cognitive } \\
\text { alteration }\end{array}$ & Hyper-phagia & $\begin{array}{l}\text { Hyper- } \\
\text { sexuality }\end{array}$ & $\begin{array}{l}\text { Triggering } \\
\text { factor }\end{array}$ & $\begin{array}{l}\text { Duration of } \\
\text { episode }\end{array}$ & $\begin{array}{l}\text { Number of } \\
\text { episodes }\end{array}$ & HLA DQB 1* & Нуро-1 \\
\hline 1 & 3 & 15 & $M$ & 13 & + & - & + & + & $10-20$ & 5 & $0301 / 0301$ & 897 \\
\hline 2 & 3 & 16 & $\mathrm{~F}$ & 15 & + & + & - & - & $1-10$ & 5 & $0301 / 0302$ & 453 \\
\hline 3 & 1 & 24 & $\mathrm{~F}$ & 16 & + & - & - & - & $4-12$ & 8 & na & 268 \\
\hline 4 & 1 & 21 & $M$ & 20 & + & + & + & + & $14-16$ & 2 & $0201 / 05$ & $221-111$ \\
\hline
\end{tabular}

Centre 1: Montpellier, France; Centre 3: Paris, France

Duration of episode $=$ extreme values, in days

na, not available

Hypo-1, level of hypocretin-1 in the CSF (pg/ml) during asymptomatic periods except for patient 4 , who had $221 \mathrm{pg} / \mathrm{ml}$ during asymptomatic periods and

$111 \mathrm{pg} / \mathrm{ml}$ during symptomatic episodes 
Table 4 CSF Hypocretin-1 in various neurological conditions

\begin{tabular}{|c|c|c|c|c|c|c|}
\hline Diagnosis & No & Centre & Sex & Age & Protein & Нypo-1 \\
\hline \multicolumn{7}{|l|}{ Neurodegenerative disease } \\
\hline Alzheimer's disease & 8 & 1,2 & $2 M, 6 F$ & $70(9.04)$ & $0.50(0.16)$ & 483.87 (85.68) (356-577) \\
\hline Parkinson's disease & 3 & 2 & $1 \mathrm{M}, 2 \mathrm{~F}$ & 71 (13) & $0.38(0.11)$ & $279.67(58.01)(221-337)$ \\
\hline Motor neurone disease & 4 & 1,2 & $2 \mathrm{M}, 2 \mathrm{~F}$ & $67(12.06)$ & $0.31(0.08)$ & 462.75 (58.53) (392-535) \\
\hline Spastic paraplegia & 3 & 1,2 & $1 \mathrm{M}, 2 \mathrm{~F}$ & $46.67(20.13)$ & $0.39(0.04)$ & 480.67 (74.78) (397-541) \\
\hline \multicolumn{7}{|l|}{ Demyelinating diseases of CNS } \\
\hline Multiple sclerosis & 14 & 1,2 & $6 \mathrm{M}, 8 \mathrm{~F}$ & $30.21(10.21)$ & $0.43(0.10)$ & $489.93(244.56)(282-1280)$ \\
\hline Devic disease & 1 & 1 & $\mathrm{~F}$ & 49 & 0.41 & 874 \\
\hline Inflammatory bowel disease & 1 & 1 & $\mathrm{~F}$ & 45 & 0.27 & 310 \\
\hline \multicolumn{7}{|l|}{ Peripheral neuropathies } \\
\hline Guillain-Barré syndrome & 2 & 1,2 & $2 \mathrm{~F}$ & $21.5(6.36)$ & $1.45(1.2)$ & 980.5 (193.04) (844-1117) \\
\hline CIDP* & $\overline{1}$ & 1 & $\vec{M}$ & 60 & 1.77 & 1333 \\
\hline Diabetic polyneuropathy & 2 & 1 & $M, F$ & $54.5(4.95)$ & $0.79(0.51)$ & 493.5 (4.95) (490-497) \\
\hline Toxic polyneuropathy & $\overline{1}$ & 1 & $M$ & 57 & 0.48 & 427 \\
\hline Idiopathic polyneuropathy & 3 & 1,2 & $3 F$ & $55(5.57)$ & $0.5(0.21)$ & $850.33(285.67)(623-1171)$ \\
\hline \multicolumn{7}{|l|}{ Vascular disease } \\
\hline Stroke & 6 & 1 & $3 M, 3 F$ & 65.67 (7.99) & $0.33(0.08)$ & 617.00 (304.71) (380-1184) \\
\hline Vascular dementia & 1 & 1 & $M$ & 70 & 0.65 & 391 \\
\hline Isolated CNS angiitis & 1 & 2 & $\mathrm{~F}$ & 48 & 0.21 & 437 \\
\hline \multicolumn{7}{|l|}{ Paraneoplastic disorders } \\
\hline Lambert-Eaton syndrome & 1 & 2 & $M$ & 63 & 0.51 & 260 \\
\hline Limbic encephalitis & 3 & 2 & $3 \mathrm{~F}$ & $63.3(14.64)$ & $0.55(0.32)$ & 448 (122.87) (312-481) \\
\hline \multicolumn{7}{|l|}{ Prion diseases } \\
\hline Creutzfeldt-Jacob disease & 5 & 2 & $5 M$ & $65.2(10.69)$ & $0.49(0.12)$ & $536.6(55.28)(491-618)$ \\
\hline \multicolumn{7}{|l|}{ Other neurological disorders } \\
\hline Normal pressure hydrocephalus & 3 & 1 & $2 M, 1 F$ & $70.67(2.88)$ & $0.43(0.08)$ & $477.67(575.77)(123-1142)$ \\
\hline Myasthenia gravis & 1 & 2 & $M$ & 40 & 0.45 & 588 \\
\hline Pseudotumour cerebri & 4 & 2 & $4 \mathrm{~F}$ & $36.25(5.56)$ & $0.30(0.09)$ & $533(33.85)(498-563)$ \\
\hline CNS neoplasm & 1 & 1 & $M$ & 72 & 0.51 & 382 \\
\hline Myoclonus-hyperexplexia & 1 & 2 & $M$ & 50 & 0.23 & 531 \\
\hline Lues & 1 & 2 & $\mathrm{~F}$ & 32 & 0.22 & 501 \\
\hline \multicolumn{7}{|c|}{ Miscellaneous neurological conditions } \\
\hline Fibromyalgia & 1 & 1 & $M$ & 49 & 0.5 & 821 \\
\hline Central vertigo & 3 & 1 & $2 M, 1 F$ & $39(20.24)$ & $0.43(0.13)$ & $695.33(457.07)(177-1280)$ \\
\hline Acute headache & 3 & 1 & $2 M, 1 F$ & $39.33(15.82)$ & $0.47(0.04)$ & $993.33(55.51)(955-1057)$ \\
\hline Epileptic seizures & 4 & 1,2 & $2 M, 2 F$ & $57.5(9.54)$ & $0.39(0.14)$ & $677.75(213.88)(534-996)$ \\
\hline Chronic sciatalgia & 1 & 1 & $\mathrm{~F}$ & 34 & 0.55 & 583 \\
\hline Cranio-cerebral trauma & 1 & 1 & $\mathrm{~F}$ & 63 & 1.59 & 583 \\
\hline Depression & 1 & 2 & $M$ & 38 & 0.31 & 595 \\
\hline Encephalitis & 1 & 2 & $\mathrm{~F}$ & 23 & 0.21 & 589 \\
\hline Other dementia & 1 & 2 & $M$ & 71 & 0.42 & 456 \\
\hline Recurrent attacks of dizziness & 1 & 2 & $M$ & 62 & 0.40 & 1259 \\
\hline
\end{tabular}

Centre 1: Montpellier, France; Centre 2: Zurich, Switzerland

Protein $=$ CSF protein expressed in $\mathrm{g} / \mathrm{ml}$

Hypo-1 = mean (SD) (range) level of hypocretin-1 in the CSF (pg/ml)

${ }^{*} \mathrm{CIDP}$, Chronic inflammatory demyelinating polyneuropathy; CNS, central nervous system

\section{RESULTS}

\section{Narcolepsy with cataplexy}

Between group comparisons of CSF hypocretin-1 levels indicated a highly significant difference $(F 6,131=16.71$, $\mathrm{p}<<0.0001)$. Reanalysis showed that narcoleptic patients with cataplexy had lower hypocretin-1 levels than any other group ( $\mathrm{p}<0.02$ to $\mathrm{p}<0.00026$ ). The overall 85 th percentile of CSF hypocretin-1 levels was at $623 \mathrm{pg} / \mathrm{ml}$ for the whole population studied, with a high level arbitrarily set at $650 \mathrm{pg} / \mathrm{ml}$.

The mean (SD) CSF hypocretin-1 level in narcoleptic patients with cataplexy was very low, $40.2(60.2 \mathrm{pg} / \mathrm{ml}$. Twenty three narcoleptic patients with cataplexy had hypocretin-1 levels $<110 \mathrm{pg} / \mathrm{ml}$, with 15 under the limit of detection, regardless of their current treatments (table 1). None had a high level $(>650 \mathrm{pg} / \mathrm{ml})$. The hypocretin- 1 level within the normal range $(271 \mathrm{pg} / \mathrm{ml})$ was found in only one patient (case 18), who had an unusual form of narcolepsy (long mean sleep latency on MSLT and negative HLADQB1*0602 typing) (table 1 ). This patient was our unique non-DQB1*0602 narcolepsy case. One narcoleptic patient with clear-cut cataplexy and positive HLA-DQB ${ }^{*} 0602$ typing had an intermediate CSF hypocretin-1 level (case 9, $126 \mathrm{pg} / \mathrm{ml}$ ); however sleep inertia either after the night-time or daytime sleep was noted and several relatives with isolated recurrent daytime naps or lapses into sleep were identified in his family (table 1). The other narcoleptic patient with cataplexy and intermediate hypocretin-1 level (case 26, $145 \mathrm{pg} / \mathrm{ml}$ ) was also a familial case with the mother affected with narcolepsy-cataplexy. Among the 26 narcoleptic probands, three reported a positive familial history of narcolepsy-cataplexy (cases 4, 17 and 26), three reported that relatives had isolated recurrent daytime naps or lapses into sleep (cases 9, 13 and 16) and all except two (case 9 and 26) had low CSF hypocretin-1 levels (table 1).

No association was found between CSF hypocretin- 1 levels and sex, body mass index, age at onset of excessive daytime sleepiness or cataplexy, age and presence of treatment at the time of lumbar puncture, Epworth sleepiness scale, ${ }^{34}$ severity of cataplexy, mean sleep latencies on MSLT and the number of sleep onset REM periods. The CSF hypocretin-1 level in a patient with status cataplecticus was under the limit of detection (case 2, table 1). The sensitivity of low CSF hypocretin-1 levels $(<110 \mathrm{pg} / \mathrm{ml})$ was $88.5 \%$ for narcoleptic patients with cataplexy, $92 \%$ for HLA-DQB $1 * 0602$ positive narcoleptic patients, and $95 \%$ for sporadic narcoleptic patients.

\section{Narcolepsy without cataplexy, other SOREMP- associated hypersomnia, idiopathic and post- traumatic hypersomnia}

The mean (SD) CSF hypocretin-1 level in narcoleptic patients without cataplexy was within the normal control range, 412.1 
(166.2) pg/ml. More precisely, eight out of the nine narcoleptic patients without cataplexy had normal CSF hypocretin-1 levels, and one patient with atypical symptoms (case 3, with long mean sleep latency on MSLT) had a low level ( 100 pg/ml) (table 2). Familial cases of narcoleptic patients without cataplexy did not differ in terms of CSF hypocretin-1 level.

Two other SOREMP-associated hypersomnia patients affected with sleep apnoea syndrome and myotonic dystrophy had normal CSF hypocretin-1 levels (table 2).

All of the patients with polysymptomatic idiopathic hypersomnia had CSF hypocretin-1 levels above $200 \mathrm{pg} / \mathrm{ml}$ with a mean (SD) level at 490.4 (307.4) pg/ml (table 2). One patient had an atypically high CSF hypocretin-1 level ( $1130 \mathrm{pg} / \mathrm{ml}$ ); the lumbar puncture was performed during a period of shift work accompanied by sleep deprivation.

The patient most severely affected with post-traumatic hypersomnia with brain lesions (as determined by MRI) had an intermediate CSF hypocretin-1 level (176 pg/ml), while the second most severely affected patient had a normal level (table 2).

\section{Patients with Kleine-Levin syndrome}

The mean (SD) CSF hypocretin-1 level in this group during the asymptomatic periods was within the normal control range, 459.8 (308.2) pg/ml (table 3). However, one patient (case 1) had a high CSF hypocretin-1 level $(897 \mathrm{pg} / \mathrm{ml})$. CSF samples were available during both asymptomatic and symptomatic periods in case 4 . Results showed a twofold decrease in hypocretin-l level during the hypersomnia $(111 \mathrm{pg} / \mathrm{ml}$ ) episode as compared to the asymptomatic period $(221 \mathrm{pg} / \mathrm{ml}$ ) (table 3). This patient was affected with both Klein-Levin syndrome and Prader-Willi syndrome.

\section{Other neurological conditions}

The mean CSF hypocretin-1 level in this group was 572.4 (268.5) $\mathrm{pg} / \mathrm{ml}$. For the 88 patients investigated, CSF hypocretin-1 levels were intermediate in three cases, and no cases had low levels (table 4). In three patients affected with normal pressure hydrocephalus, two had intermediate CSF hypocretin-1 levels ( 123 and $168 \mathrm{pg} / \mathrm{ml}$ respectively) and one had a high CSF hypocretin-1 level (1142 pg/ml). The third patient with an intermediate CSF hypocretin-1 level (177 pg/ $\mathrm{ml}$ ) was affected with central vertigo without clear organic aetiology. All other disorders, neurodegenerative disorders (Parkinson's disease, Alzheimer's disease, motor neurone disease), autoimmune inflammatory disorders (Guillain Barré syndrome, chronic inflammatory demyelinating polyneuropathy, multiple sclerosis, myasthenia gravis), paraneoplastic disorders (limbic encephalitis and Lambert-Eaton syndrome), prion diseases (sporadic Creutzfeldt-Jacob disease) had CSF hypocretin-1 levels above $200 \mathrm{pg} / \mathrm{ml}$ (table 4). In our neurological disorder population, CSF hypocretin-1 levels showed the largest variance (from 123 to $1333 \mathrm{pg} / \mathrm{ml}$, table 4). Seventeen patients with various neurological conditions had high CSF hypocretin-1 level (>650 pg/ml) and nine of those had levels above $1000 \mathrm{pg} / \mathrm{ml}$ (table 4). A weak correlation was found between CSF hypocretin-1 and CSF protein levels $(r=0.24, p<0.028)$. No association was found between sex, body mass index, or age at time of lumbar puncture and CSF hypocretin-1 levels.

\section{DISCUSSION}

The population of this study constitutes the second largest case series of CSF hypocretin-1 measurements in patients with hypersomnia and the first in patients with well-defined Kleine-Levin syndrome. The presence of a low CSF hypocretin-1 level is highly specific $(99.1 \%)$ and sensitive $(88.5 \%)$ for narcolepsy with cataplexy. We found low CSF hypocretin-1 levels in 23 out of $26(88.5 \%)$ narcoleptic patients with cataplexy. One HLA DQBI*0602 negative patient had a normal hypocretin-1 level and two patients with intermediate levels were from familial cases (major and minor phenotype), findings confirming earliest reports. ${ }^{7-11}{ }^{13}{ }^{14}$ We found normal CSF hypocretin-1 levels in eight out of nine (88.9\%) narcoleptic patients without cataplexy, a finding also reported in most previous studies. ${ }^{911} 131433$ These findings strengthen the role of cataplexy (but not its severity) in the positive diagnosis of full-blown narcolepsy and suggest a different aetiology for narcolepsy without cataplexy. However, several narcoleptic patients with clear-cut cataplexy and normal or high CSF hypocretin-1 levels have been reported as well as narcoleptic patients without cataplexy but with low CSF hypocretin-1 levels ${ }^{911} 131433$ (also observed in our population). Moreover, a dramatic decrease in the number of hypocretin-containing neurones of the hypothalamus has been reported in postmortem brains of both narcoleptic patients with and without cataplexy. ${ }^{6}$ We hypothesise a continuum of severity progressing from narcolepsy without cataplexy to narcolepsy with cataplexy with a defective hypocretin neurotransmission in rare cases.

CSF hypocretin-1 level is also closely associated with the presence of HLA DQB ${ }^{*} 0602$ allele since the only DQB1*0602 negative narcoleptic-cataplectic patient in our population had normal hypocretin levels, a finding in agreement with the literature. $^{89111433}$ In addition, among the six narcoleptic patients with relatives affected either with narcolepsycataplexy or isolated daytime naps or lapses into sleep, two had intermediate hypocretin levels, suggesting a weaker association between low hypocretin levels and familial narcolepsy. These findings confirm the earliest reports ${ }^{8} 91433$ and suggest that the $\mathrm{DQB1}{ }^{*} 0602$ allele may have a major role in conferring low or absent hypocretin neurotransmission.

Other SOREMP-associated hypersomnias included in this study ( sleep apnoea syndrome and myotonic dystrophy) were associated with normal CSF hypocretin-1 levels. These results do not support the involvement of hypocretin transmission in abnormal regulation of REM sleep in these conditions, although only a few non-narcoleptic patients with SOREMPs associated hypersomnia were investigated.

Polysymptomatic idiopathic hypersomnias were associated with normal CSF hypocretin levels as also recently reported by others..$^{911} 13$ Only one study reported a hypocretin deficiency $(<100 \mathrm{pg} / \mathrm{ml})$ in idiopathic hypersomnia. ${ }^{15}$ In our population, one atypical patient with sleep debt associated with shift work and idiopathic hypersomnia had a high CSF hypocretin level. Head trauma is another rare cause of hypersomnia in which CSF hypocretin levels were rarely investigated. ${ }^{9}$ Two head trauma patients with objective hypersomnia were included, one with an intermediate and one (less severe condition) with a normal CSF hypocretin-1 level. Although the involvement of hypocretin transmission abnormality remains uncertain in this condition, the long delay between brain injury, the occurrence of excessive daytime sleepiness, and the time of lumbar puncture may have confounded the results.

In addition to previous data, ${ }^{8} 911131433$ our results in several hypersomnia conditions strengthen the absence of CSF hypocretin-1 deficiency in the pathophysiology of most of non narcolepsy-cataplexy disorders. A hypothesis may be drawn for continuum in the pathophysiology of narcolepsy without cataplexy and idiopathic hypersomnia. ${ }^{913}$ However, a dysfunction in hypocretin-1 or 2 receptor, an ineffective signal neurotransmission or only partial hypocretin lesions without low CSF hypocretin-1 consequences cannot be definitely excluded in those disorders.

Kleine-Levin syndrome is a rare cause of hypersomnia characterised by a recurrent alteration of sleep-wake regulation and frequent abnormal feeding and sexual behaviours. ${ }^{21-23}$ 
Recent association reported with HLA DQB1*0201 together with the young age of onset, the recurrence of symptoms, and the frequent infectious precipitating factors suggest an autoimmune aetiology for Kleine-Levin syndrome. ${ }^{22}$ Since the role of hypocretin neuropeptides in both feeding behaviour and sleep-wake regulation is now well established, ${ }^{20}$ we have hypothesised a hypocretin neurotransmission abnormality in patients with Kleine-Levin syndrome. The four cases investigated during an asymptomatic interval had CSF hypocretin-1 levels in the normal range, but one patient investigated both between and within symptomatic periods had a twofold decrease in hypocretin-1 level during the symptomatic episode (this patient was also affected with Prader-Willi syndrome), suggesting a possible intermittent alteration of the hypocretin system. This finding constitutes the first report of a specific and transient decrease in CSF hypocretin-1 level in association with recurrent clinical symptoms. Recently two other patients with Kleine-Levin syndrome have been investigated during symptomatic episodes, one with intermediate ${ }^{9}$ and the other with normal ${ }^{35}$ CSF hypocretin-1 levels, but no data were available during their normal interval period. Another patient affected with Prader-Willi syndrome but without Kleine-Levin syndrome has been reported in the literature with an intermediate hypocretin level $(111 \mathrm{pg} / \mathrm{ml}),{ }^{9}$ confirming our hypothesis that episodic and state-dependent variations in hypocretin levels might occur in Kleine-Levin syndrome, either isolated or associated with Prader-Willi syndrome, and might therefore represent a biological marker of these disorders.

Finally, patients $(3.4 \%)$ with other rare neurological conditions had intermediate but never low CSF hypocretin-1 levels. Mignot et $\mathrm{al}^{9}$ reported recently that out of 194 neurological patients, 30 (15.5\%) had intermediate levels and three had low levels in various acute neuropathological conditions. Another striking finding is the intermediate hypocretin-1 level in normal pressure hydrocephalus (two out of three patients), but not in Guillain-Barré syndrome, craniocerebral trauma (without hypersomnia), paraneoplastic or encephalitis disorders as previously reported. ${ }^{89}{ }^{12} 36$ In contrast, high CSF hypocretin-1 levels occurred in several neurological conditions (including mild Guillain-Barré syndrome) and in both idiopathic hypersomnia (one patient) and Kleine-Levin syndrome (one patient). Similar results were previously reported in early-onset restless legs syndrome. ${ }^{37}$ The high CSF hypocretin-1 level $(>650 \mathrm{pg} / \mathrm{ml})$ was statistically calculated above the 85 th percentile of our overall population, but an upper limit of normal CSF is not yet known. In contrast to most patients with hypersomnia, a large variation in CSF hypocretin- 1 levels was observed in the heterogeneous neurological group although the importance of these findings remain controversial. ${ }^{8-10} 3337$ In spite of a weak correlation found between CSF protein and hypocretin-1 levels in this group, the heterogeneity in CSF hypocretin-1 levels was not explained. However, the significance of intermediate (110-200 pg/ml) and high hypocretin $(>650 \mathrm{pg} / \mathrm{ml})$ levels is still unclear and needs further investigation.

\section{Summary}

Deficient hypocretin-1 transmission is highly specific and sensitive for sporadic narcolepsy with typical excessive daytime sleepiness, cataplexy, and the HLA DQB1*0602 allele. Hypocretin ligand deficiency does not appear to be the major cause for other hypersomnias and a hypothesis could be drawn for a pathophysiological continuum between narcolepsy without cataplexy and idiopathic hypersomnia. However, a dysfunction in the hypocretin-1 or 2 receptor, or partial hypocretin lesions without low CSF hypocretin-1 consequences cannot be excluded in these disorders. The involvement of intermediate level of CSF hypocretin-1 in non-narcoleptic conditions therefore remains unclear. We may suggest a focal, transient, and immune-modulated process in the hypothalamic hypocretin system in KleineLevin syndrome, resulting in altered hypocretin neurotransmission only during symptomatic episodes.

\section{Authors' affiliations}

Y Dauvilliers, B Carlander, J Touchon, M Billiard, Service de Neurologie B, Hôpital Gui-de-Chauliac, Montpellier, France

Y Dauvilliers, M Tafti, Biochemistry and Genetics, Department of Psychiatry, Geneva, Switzerland

Y Dauvilliers, A Besset, J Touchon, INSERM E0361, Hôpital la

Colombière, Montpellier, France

C R Baumann, M Bischof, T Blatter, C L Bassetti, Neurology Clinic, University Hospital Zurich, Switzerland

M Lecendreux, Service de Psychopathologie de l'Enfant et de

I'Adolescent, Hôpital Robert Debré, Paris, France

F Maly, Institute of Clinical Chemistry, University Hospital Zurich, Switzerland

YD and MT were supported by the Geneva University Hospitals.

\section{REFERENCES}

1 Aldrich MS. Narcolepsy. Neurology 1992;42:34-43.

2 Gelineau JBE. De la narcolepsie. Gazette des Hôpitaux (Paris) 1880;53:626-8

3 Lin L, Faraco J, Li R, et al. The sleep disorder canine narcolepsy is caused by a mutation in the hypocretin (orexin) receptor 2 gene. Cell 1999;98:365-76.

4 Chemelli RM, Willie JT, Sinton CM, et al. Narcolepsy in orexin knockout mice: molecular genetics of sleep regulation. Cell 1999;98:437-51.

5 Peyron C, Faraco J, Rogers W, et al. A mutation in a case of early onset narcolepsy and a generalized absence of hypocretin peptides in human narcoleptic brains. Nat Med 2000;6:991-7.

6 Thannickal TC, Moore RY, Nienhuis R, et al. Reduced number of hypocretin neurons in human narcolepsy. Neuron 2000;27:469-74.

7 Nishino S, Ripley B, Overeem S, et al. Hypocretin (orexin) deficiency in human narcolepsy. Lancet 2000;355.1:39-40.

8 Ripley B, Overeem S, Fujiki N, et al. CSF hypocretin/orexin levels in narcolepsy and other neurological conditions. Neurology 2001;57:2253-8.

9 Mignot E, Lammers GJ, Ripley B, et al. The role of cerebrospinal fluid hypocretin measurement in the diagnosis of narcolepsy and other hypersomnias. Arch Neurol 2002;59:1553-62.

10 Dalal MA, Schuld A, Haack M, et al. Normal plasma levels of orexin A (hypocretin-1) in narcoleptic patients. Neurology 2001;56:1749-51

11 Kanbayashi T, Inove Y, Chiba S, et al. CSF hypocretin-1 (orexin-A) concentrations in narcolepsy with and without cataplexy and idiopathic hypersomnia. J Sleep Res 2002;11:91-3.

12 Kanbayashi T, Ishiguro H, Aizawa R, et al. Hypocretin-1 (orexin-A) concentrations in cerebrospinal fluid are low in patients with Guillain-Barré syndrome. Psychiatry Clin Neurosci 2002;56:273-4.

13 Bassetti C, Gugger M, Bischof $M$, et al. The narcoleptic borderland: a multimodal diagnostic approach including cerebrospinal fluid levels of hypocretin-1 (orexin-A). Sleep Med 2003;4:7-12.

14 Krahn LE, Pankratz VS, Oliver L, et al. Hypocretin (orexin) levels in cerebrospinal fluid of patients with narcolepsy: relationship to cataplexy and HLA DQB 1*0602 status. Sleep 2002;25:733-6.

15 Ebrahim IO, Sharief MK, De Lacy S, et al. Hypocretin (orexin) deficiency in narcolepsy and primary hypersomnia. J Neurol Neurosurg Psychiatry 2003;74:127-30.

16 Gibbs JW 3rd, Ciafaloni E, Radtke RA. Excessive daytime somnolence and increased rapid eye movement pressure in myotonic dystrophy. Sleep 2002;25:672-5.

17 Guilleminault C, Stoohs R, Quera-Salva MA. Sleep-related obstructive and nonobstructive apneas and neurologic disorders. Neurology 1992:42:53-60.

18 Manni R, Politini L, Nobili L, et al. Hypersomnia in the Prader-Willi syndrome: clinical-electrophysiological features and underlying factors. Clin Neurophysiol 2001;112:800-5.

19 Chervin RD, Aldrich MS. Sleep onset REM periods during multiple sleep latency tests in patients evaluated for sleep apnea. Am J Respir Crit Care Med $2000 ; 161: 426-31$

20 Willie JT, Chemelli RM, Sinton CM, et al. To eat or to sleep? Orexin in the regulation of feeding and wakefulness. Annu Rev Neurosci 2001;24:429-58.

21 American Sleep Disorders Association. International classification of sleep disorders, revised: Diagnostic and coding manual. Rochester, MN: American Sleep Disorders Association, 1997.

22 Dauvilliers Y, Mayer G, Lecendreux M. Kleine-Levin syndrome: An autoimmune hypothesis based on clinical and genetic analyses. Neurology 2002:59:1739-45

23 Billiard M. The Kleine Levin syndrome. In: Kryger MH, Roth T, Dement WC, eds. Principles and Practice of Sleep Medicine. Philadelphia: WB Saunders, 1989:377-8.

24 Billiard M, Pasquié-Magnetto V, Heckman M, et al. Family studies in narcolepsy. Sleep 1994;17:S54-9. 
25 Mignot E. Genetic and Familial aspects of narcolepsy. Neurology 1998;50(suppl 1):S16-22.

26 Harley HG, Brook JD, Rundle SA. Expansion of an unstable DNA region and phenotypic variation in myotonic dystrophy. Nature 1992;355:545-6.

27 Billiard M, Dauvilliers Y. Idiopathic hypersomnia. Sleep Med Rev 2001;5:351-60.

28 Bassetti C, Aldrich MS. Idiopathic hypersomnia. A series of 42 patients. Brain 1997; 120:1423-35.

29 Guilleminault C, Yuen KM, Gulevich MG, et al. Hypersomnia after head-neck trauma: a medicolegal dilemma. Neurology 2000;54:653-9.

30 Guilleminault C, Faull KF, Miles L, et al. Posttraumatic excessive daytime sleepiness: a review of 20 patients. Neurology 1983;33:1584-9.

31 Gunay-Aygun M, Schwartz S, Heeger S, et al. The changing purpose of Prader-Willi syndrome clinical diagnostic criteria and proposed revised criteria. Pediatrics 2001;108:E92.
32 Gau SF, Soong WT, Liu HM, et al. Kleine-Levin Syndrome in a boy with Prader-Willi syndrome. Sleep 1996;19:13-17.

33 Nishino S, Ripley B, Overeem S, et al. Low cerebrospinal fluid hypocretin (Orexin) and altered energy homeostasis in human narcolepsy. Ann Neurol 2001;50:381-8.

34 Johns MW. A new method for measuring daytime sleepiness: the Epworth sleepiness scale. Sleep 1991;14:540-5.

35 Katz JD, Ropper AH. Familial kleine-levin syndrome: two siblings with unusually long hypersomnic spells. Arch Neurol 2002;59:1959-61.

36 Overeem S, Dalmau J, Bataller L, et al. Secondary narcolepsy in patients with paraneoplastic anti-Ma2 antibodies is associated with hypocretin deficiency. J Sleep Res 2002;1 1:166-7.

37 Allen RP, Mignot E, Ripley B, et al. Increased CSF hypocretin-1 (orexin-A) in restless legs syndrome. Neurology 2002;59:639-41.

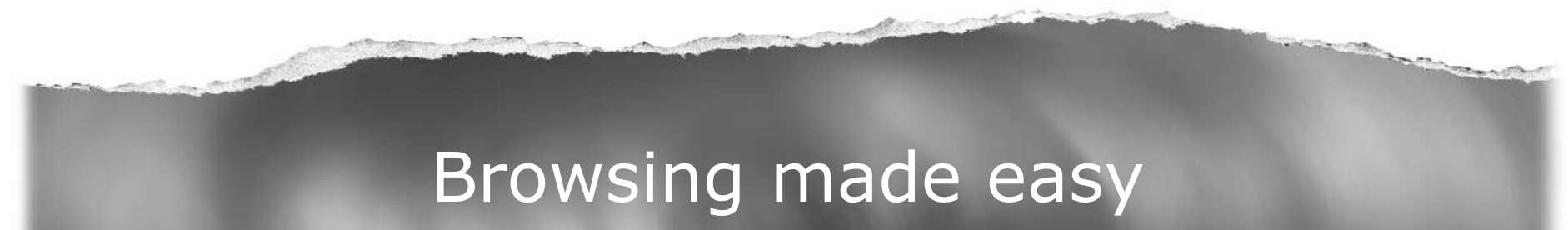

\section{Collections}

With a single click Collections allows you to find all articles that have been published in your chosen subject. Select from over 200 clinical and non-clinical topic collections and/or cross search other specialist journals, the BMJ and Cochrane Reviews

www.jnnp.com 